\title{
Desarrollo y validación de un modelo diagnóstico de resistencia antimicrobiana por $\beta$-lactamasas de espectro extendido en infecciones urinarias adquiridas en la comunidad. ¿Cómo ajustar la predicción en prevalencias variables del desenlace? La utilidad de la contracción de predictores con regresión LASSO
}

\author{
Development and validation of a diagnostic model of antimicrobial resistance by \\ extended-spectrum beta-lactamases in community-acquired urinary tract infections. \\ How to adjust the prediction in variable outcome prevalences? The usefulness of \\ contracting predictors with LASSO regression
}

\begin{abstract}
Sebastián Fernando Niño ${ }^{1,2}$, Natalia Maldonado ${ }^{4}$, Julio Biojo ${ }^{1}$, Rosa María Ospina ${ }^{1}$ Pamela Velásquez¹, Hernán Aguirre², Gilma Hernández², Fabio González y Héctor Iván García
\end{abstract}

\footnotetext{
${ }^{1}$ Comité de Infecciones Clínica SaludCoop Medellín Colombia.

2 Departamento de Epidemiología Clínica, Universidad de Antioquia. Colombia

${ }^{3}$ MindLab, Departamento de Ingeniería, Universidad Nacional de Colombia.

${ }^{4}$ Grupo Germen, Medellín Antioquia. Colombia.

Clínica SaludCoop Medellín Colombia. Enero 2012- diciembre 2016.
}

Los autores declaran ausencia de conflictos de interés.

Financiación: recursos propios de los autores.

Recibido: 4 de abril de 2020 (Segunda versión: 9 de agosto de 2020) / Aceptado: 26 de octubre de 2020

\section{Resumen}

Introducción: Para el caso de infección urinaria adquirida en la comunidad la identificación de enterobacterias con $\beta$-lactamasas de espectro extendido (BLEE) puede optimizar las estrategias de tratamiento, control y seguimiento; sin embargo, el efecto de prevalencias variables de este patrón de resistencia ha afectado la validez externa de este tipo de modelos. Objetivo: Desarrollar un modelo predictor diagnóstico que ajuste el error de predicción en prevalencias variables utilizando la regresión LASSO. Métodos: Se diseñó un modelo predictor diagnóstico de infección urinaria adquirida en la comunidad por enterobacterias productoras de BLEE. Se empleó un estudio de corte transversal, tanto para la construcción como para la validación. Para evaluar el efecto de la prevalencia variable del desenlace, la validación se realizó con población en la que la proporción de aislados con este mecanismo de resistencia fue menor, los participantes fueron pacientes adultos que consultaron a servicios de urgencias de dos instituciones hospitalarias de mediano nivel de complejidad de la ciudad

\begin{abstract}
Background: In the case of community-acquired urinary tract infection, the identification of Enterobacteriaceae with extended spectrum beta-lactamases (ESBL) can optimize treatment, control and follow-up strategies, however the effect of variable prevalences of this resistance pattern has affected the external validity of this type of models. Aim: To develop a diagnostic predictive model that adjusts the prediction error in variable prevalences using the LASSO regression. Methods: A diagnostic predictive model of communityacquired urinary tract infection by infection by ESBL producing Enterobacteriaceae was designed. A cross-sectional study was used for both construction and validation. To assess the effect of the variable prevalence of the outcome, the validation was performed with a population in which the proportion of isolates with this resistance mechanism was lower, the participants were adult patients who consulted the emergency services of two medium-level hospital institutions. complexity of the city of Medellin. To adjust for the effect of
\end{abstract}


de Medellín. Para ajustar el efecto de un medio ambiente con menor proporción de resistencia antimicrobiana, utilizamos la contracción de predictores por regresión LASSO. Resultados: Se incluyeron 303 pacientes para la construcción del modelo, se evaluaron seis predictores y la validación se realizó en 220 pacientes. Conclusión: El modelo ajustado con regresión LASSO favoreció la validez externa del modelo en poblaciones con proporción de aislados productores de BLEE en urocultivo de pacientes ambulatorio entre 11 y $16 \%$. Este estudio brinda criterios para un aislamiento temprano cuando los predictores están presentes en poblaciones con proporciones de resistencia en urocultivos ambulatorios cercanas a $15 \%$ y propone una metodología para ajuste de error en el diseño de modelos de predicción en resistencia antimicrobiana

Palabras clave: modelos logísticos; resistencia a $\beta$-lactámicos; infección del tracto urinario.

\section{Introducción}

U no de los problemas médicos de mayor interés relacionados con infecciones por enterobacterias es la infección del tracto urinario (ITU). Desde que se describieron $\beta$-lactamasas con capacidad destruir cefalosporinas de tercera generación a mediados de la década de los 80 , este tipo de resistencia se ha convertido en un problema de salud pública global ${ }^{1}$. El panorama del tratamiento de infecciones adquiridas en la comunidad puede complicarse en la medida que los patrones de resistencia vistos de manera usual en el ambiente hospitalario se diseminan en la comunidad. Para la ciudad de Medellín (Colombia) según datos del grupo germen (http://www. grupogermen.org) la proporción de aislados de enterobacterias con $\beta$-lactamasas espectro extendido (BLEE) para ITU de pacientes atendidos ambulatoriamente ha venido aumentando de manera progresiva en los últimos años, para situarse en $12 \%$ para Escherichia coli y 17\% para Klebsiella pneumoniae.

La identificación de pacientes con alto riesgo de infecciones por microorganismos resistentes pudiera ser de ayuda para el planeamiento de estrategias terapéuticas y de control; sin embargo, existe una brecha de incertidumbre entre el abordaje terapéutico inicial y el momento en que se disponen los resultados del cultivo. El empleo de tratamiento antimicrobiano empírico inefectivo se ha relacionado con aumento en la mortalidad y de estancia hospitalaria, lo cual pudo demostrarse en una revisión sistemática publicada en el año $2015^{3}$.

El desarrollo de modelos de predicción en resistencia antimicrobiana se ha constituido en una estrategia estadística para identificar los casos con mayor probabilidad de tener infecciones por microorganismos resistentes ${ }^{14}$. En el año 2019, Mohd Sazlly y cols., publicaron una revisión sistemática acerca de modelos de predicción o colonización para enterobacterias productoras de $\mathrm{BLEE}^{2}$. Gran parte de las limitaciones expuestas en esta revisión fue la ausencia de validación externa en la mayoría de trabajos, lo cual crea una importante limitación en la generalización de las conclusiones. Consideramos un serio problema dentro de los procesos de validación externa el efecto de prevalencias an environment with a lower proportion of antimicrobial resistance, we used the contraction of predictors by LASSO regression. Results: 303 patients were included for the construction of the model, six predictors were evaluated and validation was carried out in 220 patients. Conclusion: The adjusted model with LASSO regression favored the external validity of the model in populations with a proportion of ESBL producing isolates in urine culture of outpatients between 11 and $16 \%$. This study provides criteria for early isolation when predictors are present in populations with proportions of resistance in ambulatory urine cultures close to $15 \%$ and proposes a methodology for the adjustment of errors in the design of prediction models for antimicrobial resistance.

Keywords: logistic models; beta-lactam resistance; urinary tract infections.

variables de resistencia BLEE, lo cual puede afectar negativamente el desempeño de los modelos por efecto del sobreajuste.

El objetivo de este estudio fue desarrollar y validar un modelo predictor diagnóstico de resistencia antimicrobiana por BLEE en enterobacterias causantes de infección urinaria adquirida en la comunidad en pacientes adultos, y ajustar su desempeño para poblaciones con prevalencias variables de resistencia por BLEE.

\section{Metodología}

\section{Desarrollo}

Se realizó un estudio de corte transversal, tanto para el desarrollo como para la validación externa del modelo. La población objetivo del estudio fueron pacientes adultos con ITU adquirida en la comunidad, confirmada con un urocultivo positivo. Las instituciones participantes atienden población general y en los últimos años, la proporción de aislados provenientes de urocultivos ambulatorios con perfil de resistencia BLEE ha mostrado una tendencia en aumento, cerca de 1,7\% por año, siendo aproximadamente de $16 \%$ para el 2016. La medición de las variables seleccionadas como predictores se realizó teniendo en cuenta la presencia o ausencia de las mismas hasta tres meses antes del ingreso del paciente al servicio de urgencias.

Los criterios de inclusión fueron individuos adultos con ITU adquiridas en la comunidad, confirmada con urocultivo positivo, que asistieron a los servicios de urgencias de las clínicas participantes entre el 1 de enero de 2012 y el 31 de diciembre de 2016. Se excluyeron del estudio los pacientes que tuvieran antecedentes de tratamiento con carbapenémicos en los últimos 15 días, pacientes con bacteriuria asintomática, ingreso a urgencias por derivación desde otra institución, o más de un proceso séptico en evolución al momento del ingreso al servicio a urgencias.

El desenlace de interés fue la presencia o ausencia de un microrganismo con patrón de resistencia BLEE en urocultivo por sistema VITEK 2 Compact ((bioMerieux, Inc., Hazelwood, MO, USA). 
mejor subconjunto, basándonos en criterio de información bayesiano se incluyeron los conjuntos de variables con el mejor ajuste dándole prioridad al modelo más parsimonioso. Usamos la regresión logística multivariable con un valor de $\mathrm{P}$ mayor que 0,05 para la eliminación de variables no significativas en el modelo. Debido a las restricciones por tamaño de muestra no se realizó evaluación de interacciones.

\section{Validación}

Se evaluó la validez interna realizando un proceso de re muestreo (dejar uno afuera), se estimaron nuevamente los coeficientes y, a fin de disminuir el efecto del sobreajuste, se realizó regresión contraída con LASSO. El desempeño del modelo se evaluó según la capacidad de discriminación y calibración con área bajo la curva ROC y estadístico de Hosmer Lemeshow. La validez interna del modelo se realizó utilizando el algoritmo del modelo de regresión contraída. Se definió el punto de la curva ROC que brindaba un mejor desempeño en términos de sensibilidad y especificidad, según las probabilidades predichas por el modelo.

Realizamos el proceso de validación externa utilizando datos de años anteriores en los que la proporción de aislados BLEE en orina fue más baja, con el fin de evaluar el comportamiento diagnostico en población con proporción de desenlaces más bajos. Los resultados de la validación externa nos llevaron a actualizar los modelos. Ajustamos los coeficientes regresión de los modelos de predicción por LASSO en la muestra de validación externa con una proporción de desenlaces menor y evaluamos el desempeño del modelo según la capacidad de discriminación y calibración con área bajo la curva ROC y estadístico de Hosmer Lemeshow.

Finalmente, se derivó una regla de diagnóstico a partir de los coeficientes multivariables reducidos, redondeados, para estimar la probabilidad de presencia de patrón de resistencia BLEE en el urocultivo.

\section{Resultados}

Del total de urocultivos positivos del año 2016 (n: 526) se seleccionó una muestra aleatoria de 409 casos, de los cuales 303 cumplieron con criterios de inclusión y no de exclusión; con dicha muestra se desarrolló la construcción del modelo (Figura 1).

Las características de los participantes en la muestra de desarrollo se resumen en la Tabla 1.

La proporción de datos faltantes para cada una de los predictores no supero el 5\% en variable alguna, las características de los casos con datos faltantes fueron las mismas de los casos sin datos faltantes, se realizó imputación múltiple teniendo como método de imputación el mismo método de análisis de datos. 
Utilizando la totalidad de la muestra de desarrollo, seleccionamos el modelo con menor AIC y BIC, modelo que incluía las variables edad, antecedentes de ser usuario de catéter vesical por más de $24 \mathrm{~h}$ en los últimos tres meses previos al ingreso a urgencias y el número de ciclos antimicrobianos a los cuales el paciente estuvo expuesto. Evaluamos el factor de inflación de varianza para cada una

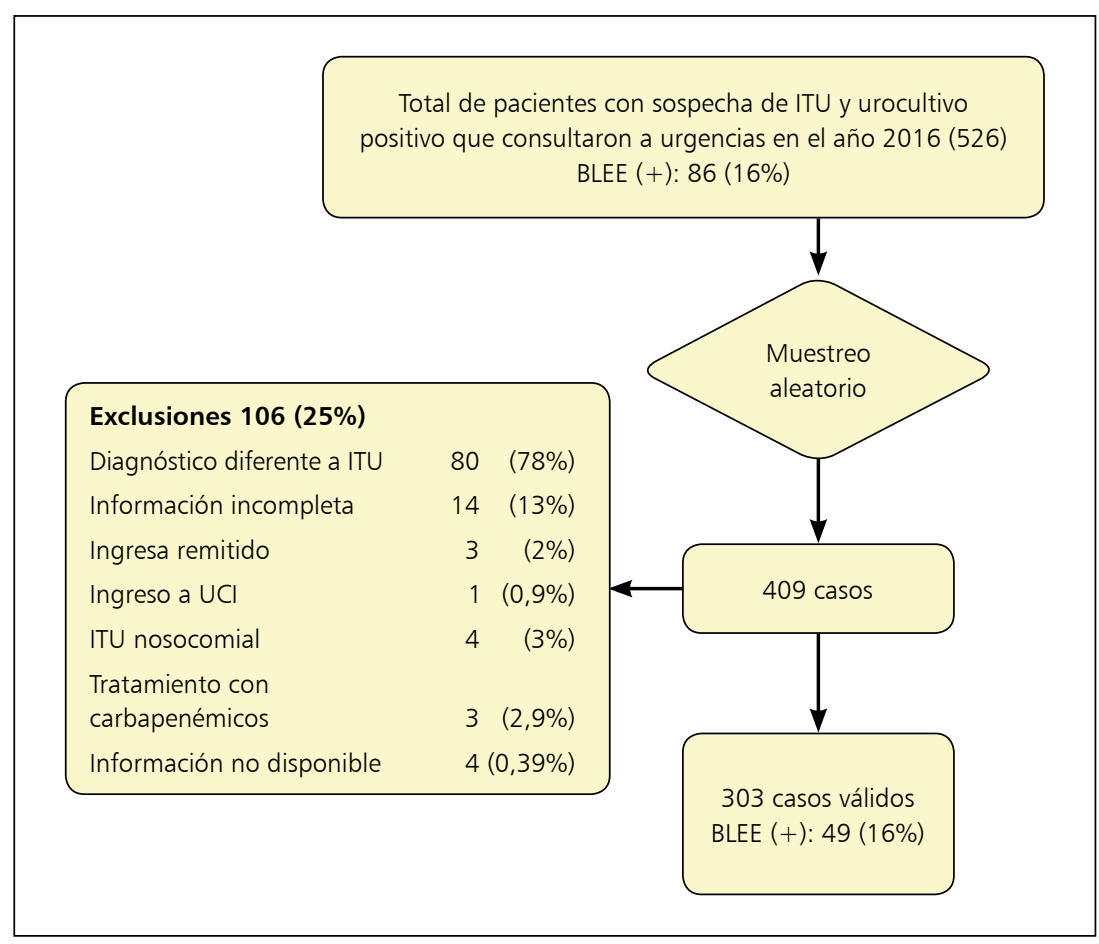

Figura 1. Flujorama de pacientes participantes en el desarrollo del modelo. de las variables incluidas en el modelo final encontrando todos los valores para cada variable cercanos a 1,0.

Para el modelo completo con sus coeficientes e intersección realizamos un proceso de validación utilizando el método de Leave One Out, fraccionando la muestra de desarrollo en 10 sub-muestras en las cuales se realizaron análisis iterativos dejando una de las fracciones afuera. Se completó el proceso de validación interna por medio de regresión contraída LASSO a fin de optimizar el comportamiento de predicción. El modelo final con sus coeficientes, intercepto y grado de penalización tras la regresión LASSO se especifica en la Tabla 2.

Realizamos test de calibración a fin de evaluar si el modelo final ajusta a los datos, el test de bondad de ajuste de Hosmer Lemeshow comparó por deciles los resultados predichos con los observados, reportando un valor $\mathrm{p}$ de 0,71 por lo cual se acepta la hipótesis nula y se determina que el modelo ajusta de manera apropiada. La capacidad de discriminación se evaluó con área bajo la curva ROC la cual reportó un valor de 0,85

Las gráficas de diagnóstico del modelo permitieron identificar tres valores atípicos, se revisó el proceso de registro, digitación, y fuente de información sin encontrar errores por lo cual se dejaron finalmente dichos valores en el modelo.

Se evaluó el comportamiento predictor para tres grupos de riesgo (bajo, medio, y alto), se pudo establecer que el grupo de pacientes identificados con una probabilidad menor de $20 \%$ para el desenlace de interés tuvo un valor predictor negativo de $93 \%$, con una sensibilidad de $69 \%$ y especificidad de $86 \%$. Los pacientes con una probabilidad mayor de $50 \%$ tuvieron un valor predictor positivo de $71 \%$ con una razón de verosimilitud positiva de 12 con

\begin{tabular}{|c|c|c|c|c|}
\hline Variable & $\begin{array}{l}\text { Urocultivo positivo } \\
\text { para microorganismo } \\
\text { productor de BLEE } \\
n=49(\%)\end{array}$ & $\begin{array}{l}\text { Urocultivo positivo } \\
\text { sin patrón de } \\
\text { resistencia BLEE } \\
n=254(\%)\end{array}$ & $\begin{array}{c}\text { Total } \\
n=303(\%)\end{array}$ & $\begin{array}{c}\text { Valores perdidos } \\
\text { (\%) }\end{array}$ \\
\hline Edad media en años \pm DS & $64 \pm 19$ & $50 \pm 20,9$ & $52 \pm 21$ & $0(0,0 \%)$ \\
\hline Sexo femenino (\%) & $26(53 \%)$ & $193(75 \%)$ & $219(72 \%)$ & $0(0,0 \%)$ \\
\hline Hospitalización previa (\%) & $17(34 \%)$ & $23(9 \%)$ & $40(13 \%)$ & $1(0,3 \%)$ \\
\hline Días de hospitalización media en días \pm DS & $3,14 \pm 0$ & $0,63 \pm 0$ & $1,0 \pm 0$ & $5(1,6 \%)$ \\
\hline Antimicrobiano últ imos 3 meses & $30(63 \%)$ & $52(20 \%)$ & $82(27 \%)$ & $4(1 \%)$ \\
\hline Número de ciclos antimicrobianos media \pm DS & $1,0 \pm 0$ & $0,2 \pm 0$ & $0,3 \pm$ & $10(3 \%)$ \\
\hline Patología urológica & $23(47 \%)$ & $44(10 \%)$ & $67(20 \%)$ & $1(0,3 \%)$ \\
\hline Patología prostática & $11(22 \%)$ & $18(7 \%)$ & $29(9 \%)$ & $0(0,0 \%)$ \\
\hline Antecedentes de IVU & $22(44 \%)$ & $43(16 \%)$ & $65(21 \%)$ & $1(0,3 \%)$ \\
\hline Catéter vesical & $10(20 \%)$ & $7(2 \%)$ & $17 \quad(5 \%)$ & $0(0,0 \%)$ \\
\hline Aislado previo productor de BLEE & $4(8 \%)$ & $3(1 \%)$ & $7(2 \%)$ & $2(0,7 \%)$ \\
\hline
\end{tabular}


una sensibilidad de $30 \%$ y una especificidad de $97 \%$ para el desenlace estudiado. Este análisis permitió establecer que la presencia de predictores en todos los grupos de riesgo no lograba establecer una adecuada sensibilidad; sin embargo, su ausencia daba un valor predictor negativo muy elevado (Tabla 3).

La validación externa se realizó tomando dos muestras aleatorias de poblaciones en las que la proporción de aislados con el desenlace de interés fue significativamente menor. Se seleccionó una muestra aleatoria de 180 pacientes con una proporción de aislamiento de enterobacterias en urocultivo de $9 \%$; después de las exclusiones se lograron 112 casos con 11 desenlaces de interés positivos, la segunda muestra de validación se realizó tomando una muestra aleatoria de 147 casos de una población donde la proporción de enterobacterias con resistencia BLEE fue de $14 \%$. Luego de las exclusiones se obtuvieron 118 casos válidos, con 13 desenlaces para una proporción de aislados productores de BLEE de $11 \%$.

Evaluamos el desempeño diagnóstico del modelo penalizado con LASSO en las dos muestras de validación externa, se compararon los deciles predichos con los observados, por estadístico de Hosmer Lemeshow y se pudo establecer que el modelo ajusta de manera apropiada para las dos poblaciones con un valor de p para ambos casos mayor de 0,05 , se evaluó la capacidad de discriminación con área bajo la curva ROC por encima de 0,8 .

Se realizó un ajuste adicional al modelo teniendo en cuenta un grado de penalización de coeficientes dependiendo la proporción de diseminación del patrón de resistencia en cada población. A medida que la proporción de aislados en la muestra disminuye, se evidenció cómo el grado de penalización aumentaba de manera exponencial, el desempeño diagnóstico para identificar el desenlace se afectó, disminuyendo la exactitud del modelo a medida que la prevalencia del desenlace se aleja de la que se empleó para la construcción del modelo, el desempeño de los predictores también se vio afectado al punto de excluir del modelo el predictor "edad" cuando la proporción de desenlaces bajó a 9\% (Tabla 4). Esto pone de manifiesto que el modelo muestra serias limitaciones cuando es utilizado en poblaciones donde la proporción de aislados productores de BLEE en orina es menor a $9 \%$.

Como regla de aplicación clínica, tomamos a consideración las características operativas en términos de sensibilidad y valor predictor negativo. Recomendamos aplicar este modelo en instituciones donde la proporción de aislados con patrón de resistencia BLEE en urocultivos ambulatorios esté entre 12 y $16 \%$. Para instituciones con una proporción de aislados menores a $10 \%$ el efecto de los predictores es mínimo y no se recomienda su uso. Dada la simplicidad de los predictores, y con el fin de dar practicidad a la aplicación del modelo, recomendamos el aislamiento preventivo y toma de urocultivo en todos los

\begin{tabular}{|c|c|c|c|}
\hline \multirow[t]{2}{*}{ Variable } & \multicolumn{3}{|c|}{ Modelo de validación cruzada } \\
\hline & Coeficiente & Error Std & Valor $p$ \\
\hline Intercepto & $(-4,08)$ & 0,63 & $(0,001)$ \\
\hline Número de ciclos antimicrobianos & 1,3 & 0,24 & $(0,001)$ \\
\hline Edad & 0,019 & 0,009 & $(0,04)$ \\
\hline Usuario de catéter vesical & 1,96 & 0,6 & $(0,001)$ \\
\hline Patología urológica & 1,04 & 0,38 & $(0,07)$ \\
\hline Deviance null & \multicolumn{3}{|l|}{268} \\
\hline Residual deviance & \multicolumn{3}{|l|}{189} \\
\hline AIC & \multicolumn{3}{|l|}{199} \\
\hline Exactitud / DE exactitud & 0,86 & \multicolumn{2}{|l|}{0,042} \\
\hline Sensibilidad / IC (95\%) & 0,36 & \multicolumn{2}{|l|}{$0,22-0,51$} \\
\hline Especificidad / IC (95\%) & 0,97 & \multicolumn{2}{|l|}{$0,95-0,99$} \\
\hline VPP / IC (95\%) & 0,72 & \multicolumn{2}{|l|}{$0,52-0,91$} \\
\hline VPN / IC (95\%) & 0,88 & \multicolumn{2}{|l|}{$0,84-0,92$} \\
\hline LR+ & 13 & \multicolumn{2}{|l|}{$5,88-30$} \\
\hline LR- & 0,65 & \multicolumn{2}{|l|}{$0,53-0,81$} \\
\hline
\end{tabular}

Tabla 3. Características operativas del modelo estratificando para grupos de bajo riesgo (probit 0.2)

\begin{tabular}{llcc|}
\hline & Valor & \multicolumn{2}{c|}{ IC (95\%) } \\
\hline Sensibilidad (\%) & 69,39 & 55,46 & 83,31 \\
\hline Especificidad (\%) & 86,22 & 81,78 & 90,66 \\
\hline Índice de validez (\%) & 83,5 & 79,15 & 87,84 \\
Valor predictor + (\%) & 49,28 & 36,75 & 61,8 \\
\hline Valor predictor - (\%) & 93,59 & 90,24 & 96,94 \\
Prevalencia (\%) & 16,17 & 11,86 & 20,48 \\
\hline Índice de Youden & 0,56 & 0,42 & 0,69 \\
Razón de verosimilitud + & 5,04 & 3,52 & 7,21 \\
\hline Razón de verosimilitud - & 0,36 & 0,23 & 0,54 \\
\hline
\end{tabular}

pacientes que presenten al menos 3 de los 4 predictores definidos (edad mayor a 65 años, tener antecedentes de patología urológica, haber estado expuesto a un ciclo antimicrobiano o más en los últimos tres meses, o haber sido usuario de catéter vesical por más de $24 \mathrm{~h}$ en los últimos tres meses) o que tengan exposición a dos ciclos de antimicrobianos o más en los últimos tres meses, acompañado de cualquiera de los otros predictores. En ausencia de todos dichos predictores, la probabilidad de 


\section{_—}

\begin{tabular}{|c|c|c|c|c|}
\hline Variable & $\begin{array}{l}\text { Modelo de validación cruzada } \\
\text { Coeficiente }\end{array}$ & $\begin{array}{l}\text { Modelo LASSO } \\
\text { Coeficiente }\end{array}$ & $\begin{array}{l}\text { Modelo LASSO } 11 \% \\
\text { Coeficiente }\end{array}$ & $\begin{array}{l}\text { Modelo LASSO 9\% } \\
\text { Coeficiente }\end{array}$ \\
\hline Lambda & 0 & 0,012 & 0,037 & 0,074 \\
\hline Intercepto & $(-4,08)$ & $(-3,3)$ & $(-2,6)$ & $(-2,05)$ \\
\hline Número de ciclos antimicrobianos & 1,3 & 1,2 & 0,99 & 0,75 \\
\hline Edad & 0,019 & 0,012 & 0,003 & 0 \\
\hline Usuario de catéter vesical & 1,96 & 1,6 & 1,19 & 0,59 \\
\hline Patología urológica & 1,04 & 0,7 & 0,45 & 0,32 \\
\hline
\end{tabular}

infección con patrón de resistencia BLEE es mínima, recomendamos entonces tratamiento empírico según recomendaciones locales y seguimiento clínico.

\section{Discusión}

Presentamos un modelo diagnóstico para identificar la presencia de resistencia mediada por BLEE en urocultivo de pacientes con ITU adquirida en la comunidad.

El proceso de validación externa reportó cómo el rendimiento diagnóstico del modelo se afecta a medida que el patrón de resistencia en dicha población se encuentra en menor proporción. Este hallazgo es concordante con el mayor problema que han presentado este tipo de modelos y es cómo el error de predicción aumenta de manera sustancial cuando se aplica a una población diferente en la que se aplicó el modelo original ${ }^{12}$.

El fenómeno relacionado con este aumento en el error de predicción en la muestra de validación externa obedece a un sobreajuste (overfitting) del modelo original a los datos con los cuales se desarrolló dicho modelo. Para evitar dicho fenómeno se utilizan técnicas de penalización o regresión contraída como la regresión LASSO.

Presentamos un método de ajuste a los coeficientes de regresión utilizando penalización por LASSO, el cual permitió identificar un patrón de contracción de coeficientes a medida que la proporción de aislados disminuía, lo cual eventualmente permitiría disminuir los errores de predicción en modelos diagnósticos de enfermedades infecciosas en los que se pretenda validar desenlaces medioambientales y así mismo poder establecer cuándo es conveniente descartar la utilización del modelo. Para este caso identificamos cómo la utilidad del modelo no fue adecuada en poblaciones con proporciones de aislamientos BLEE menores a 9\%.

Para nuestro estudio, la ausencia de predictores: catéter vesical por más de 24 h, y exposición a ciclos antimicrobianos en los últimos tres meses, patología urológica y una edad menor de 65 años brindan un elevado valor predictor negativo para el patrón de resistencia BLEE en el urocultivo del caso referente. La presencia de al menos 3 de los 4 predictores indicaría, además del inicio de tratamiento antimicrobiano empírico, la necesidad de realizar aislamiento preventivo del paciente y el análisis microbiológico correspondiente para confirmar la presencia de patrón de resistencia BLEE.

Recomendamos en el diseño de modelos diagnósticos de enfermedades infecciosas especificar características descriptivas de la población como proporciones de aislados con determinado atributo para de esta manera poder establecer el grado de similitud o con el modelo original y de esta manera anticipar el rendimiento diagnostico esperado. En todos se debe incluir un método de penalización con el propósito de disminuir el efecto del sobreajuste y mejorar de esta manera la validez externa del modelo.

\section{Referencias bibliográficas}

1.- Maldonado N A, Múnera M I, López J A, Sierra P, Robledo C G, Robledo J, et al. Tendencias de la resistencia a antibióticos en Medellín y municipios del Área Metropolitana entre 20072012: resultados de seis años de vigilancia. Biomédica. 2014; 34 (3): 433-46. 8. doi: https:// doi.org/10.7705/biomedica.v34i3.1658

2.- Mohd Sazlly Lim S, Wong P L, Sulaiman H, Atiya N, Hisham Shunmugam R, Liew
S M. Clinical prediction models for ESBLEnterobacteriaceae colonization or infection: a systematic review. J Hosp Infect. 2019; 102(1): 8-16. doi: 10.1016/j.jhin.2019.01.012

3.- Raman G, Avendano E, Berger S, Menon $\mathrm{V}$. Appropriate initial antibiotic therapy in hospitalized patients with gram-negative infections: systematic review and metaanalysis. BMC Infect Dis. 2015; 1-11. doi: 10.1186/s12879-015-1123-5.

4.- Sotto A, Boever C M D E, Fabbro-peray P,
Gouby A, Sirot D, Jourdan J. Risk factors for antibiotic-resistant Escherichia coli isolated from hospitalized patients with urinary tract infections: a prospective study. J Clin Microbiol. 2001; 39(2): 438-44. PMID: 11158087.

5.- Trecarichi EM, Cauda R, Tumbarello $M$. Detecting risk and predicting patient mortality in patients with extended-spectrum $\beta$-lactamase-producing Enterobacteriaceae bloodstream infections. Future Microbiol 2012; 
7(10): 1173-89. doi: 10.2217/fmb.12.100.

6.- Tumbarello M, Trecarichi E M, Bassetti M, De Rosa F G, Spanu T, Di Meco E, et al. Identifying patients harboring extendedspectrum-beta-lactamase- producing Enterobacteriaceae on hospital admission: derivation and validation of a scoring system. Antimicrob Agents Chemother. 2011; 55 (7): 3485-90. doi: 10.1128/AAC.00009-11.

7.- Collins G S, Reitsma J B, Altman D G, Moons K G M. Transparent reporting of a multivariable prediction model for individual prognosis or diagnosis (TRIPOD): The TRIPOD Statement. Eur J Clin Invest 2017; 162 (1). doi: 10.1111/eci.12376.

8.- Waljee A K, Higgins P D R, Singal A G. Clinical / narrative review. A primer on predictive models. Clin Transl Gastroenterol 2014; 5 (1): e44-4. doi: 10.1038/ctg.2013.19.

9.- Aguilar-Duran S, Horcajada J P, Sorlí L, Salvad M, Grau S, Gómez J, et al. Community-onset healthcare-related urinary tract infections: comparison with community and hospitalacquired urinary tract infections. J Infect Dis 2012; 64 (5): 478-83. doi:https://doi. org/10.1016/j.jinf.2012.01.010.

10.- Lee D S, Lee C B, Lee S J. Prevalence and risk factors for extended spectrum beta-lactamaseproducing uropathogens in patients with urinary tract infection. Korean J Urol 2010; 51 (7): 492-7. doi: 10.4111/kju.2010.51.7.492.

11.- Aviles C, Betancour P, Velasco C L, Godoy R, Barthel R, Martínez F. Factors associated with extended -spectrum betalactamases- producing organisms among patients with urinary tract infections: a prospective cohort study. Rev Chilena Infectol 2016; 33 (6): 628-34. doi: 10.4067/s0716-10182016000600004.

12.- Slekovec C, Bertrand X, Leroy J, Faller J $\mathrm{P}$, Talon D, Hocquet D. Identifying patients harboring extended-spectrum- $\beta$-lactamaseproducing Enterobacteriaceae on hospital admission is not that simple. Antimicrob Agents Chemother 2012; 56 (4): 2218-9. doi: 10.1128/
AAC.06376-11

13.- Lee C H, Chu F Y, Hsieh C C, Hong M Y, Chi C H, K O W C, et al. A simple scoring algorithm predicting extendedspectrum $\beta$-lactamase producers in adults with community-onset monomicrobial Enterobacteriaceae bacteremia: Mattersof frequent emergency department users. Medicine (Baltimore) 2017; 96 (16): e6648. doi: 10.1097/ MD.0000000000006648.

14.- Oshthoff M, McGuinness S L, Wagen A Z, Eisen D P. Urinary tract infections due to extended-spectrum beta-lactamase-producing Gram-negative bacteria: identification of risk factors and outcome predictors in an Australian tertiary referral hospital. Intern J Infect Dis 2015; 34: 79-83. 10.1016/j.ijid.2015.03.006.

15.- Reilly B M, Evans A T. Translating clinical research into clinical practice: impact of using prediction rules to make decisions. Ann Intern Med. 2006; 144: 201-9. doi: 10.7326/00034819-144-3-200602070-00009. 